\title{
Diagnostic Accuracy of Mri/Mra in Detecting Subscapularis Tears of Patients Scheduled For Rotator Cuff Surgery: A Mini-Systematic Review
}

\author{
Kandel M*, Stibane A and Kandel E \\ Department of Physiotherapy, Physiotherapy Institute, Switzerland
}

Received: February 13, 2018; Published: February 20, 2018

*Corresponding author: Michel Kandel, Physiotherapy-Institute, Staatsstrasse 46, 9463 Oberriet, Switzerland, Email: kandel.physio@bluewin.ch

\begin{abstract}
Background: Subscapularis tears often occur in combination with various shoulder problems. Untreated, they can lead to persisted symptoms even after rotator cuff surgery. Clinical tests, magnetic resonance imaging (MRI) and magnetic resonance arthrography (MRA) are being used to evaluate subscapularis integrity. Reviews regarding publications till 2012, however, show poor sensitivity in detecting subscapularis tears prior to surgery. Recent technological development might have led to improvement.
\end{abstract}

Method: A mini systematic review of the PubMed database of the last 5 years was made. Studies comparing MRI/MRA (index tests) to open- and arthroscopic surgical findings (reference test) and recording different types of subscapularis tears were included. Methodological appraisal was performed using QUADAS 2.

Results: In four retrospective studies, comprising 811 patients who underwent shoulder surgery, 264 subscapularis tears were identified by arthroscopy (prevalence $32.6 \%$ ). The sensitivity for detecting full thickness tears varied from $93 \%$ to $100 \%$. The sensitivity for detecting partial tears varied from $54 \%$ to $81 \%$. There were high concerns regarding the index test bias and applicability in 3 of 4 studies. The lack of consensus in the classification of subscapularis tears led to a high level of heterogeneity in these studies and made it impossible to pool data.

Conclusion: Despite the technological improvements in the last five years, the sensitivity of detecting subscapularis tears in patients scheduled for rotator cuff surgery remains challenging. Only diagnoses of bigger subscapularis tears reach a good accuracy in MRI/MRA.

Abbreviations: MRI: Magnetic Resonance Imaging; MRA: Magnetic Resonance Arthrography; TP: True Positives; TN: True Negatives; FP: False Positives; FN: False Negatives

\section{Introduction}

Subscapularis tears are seldom isolated [1] and often occur in combination with various shoulder problems like labrum-, biceps-, or other rotator cuff tears [2-4]. Although often underestimated, the prevalence of subscapularis tendon tears in patients with shoulder pain is about 27-37\% [5-7]. Clinical tests, which have been described to assess the subscapularis function, are the lift-off test, belly-press test, internal rotation lag sign, Napoleon test, and bear-hug test. The accuracy of these tests in detecting subscapularis tears is limited. Despite good specificity (reaches $91-100 \%$ ) the sensitivity of these tests is poor (between 18 - 65\%) [5,6,8]. Thus, approximately more than $40 \%$ of the subscapularis tears will be missed as only massive tears can be detected by clinical tests $[8,9]$. Failure to treat a subscapularis tear, even in supraspinatus tendon repair, can lead to persisted symptoms and failure of the portion of the cuff that was repaired [10-14].

Medical imaging technologies, such as magnetic resonance imaging (MRI) and magnetic resonance arthrography (MRA) are being used as an add-on test in the diagnostic pathway to provide useful information to establish a proper treatment plan [15]. The systematic reviews regarding publications until 2012, however, show a good accuracy for full thickness tears, but low to moderate sensitivity for partial tendon tears in rotator cuff lesions $[3,15,16]$ and in subscapularis in particular $[17,18]$. With respect to the technological improvement [19], the question rises, if MRI or MRA can accurately identify subscapularis tears and prevent inefficient treatment of shoulder problems in patients for whom surgery 
is being considered. The purpose of this systematic review is to evaluate research over the last five years of the overall accuracy and sensitivity of MRI/MRA in detecting subscapularis tears and to confirm its position as an add-on test in the diagnostic pathway in patients with shoulder problems for whom surgery is being considered.

\section{Methods}

\section{Types of Studies}

All studies of diagnostic accuracy that compared MRI/MRA (index test) with open surgery and/or arthroscopy (reference test) in patients with suspected rotator cuff pathology were included. Because of technical improvement and the existence of systematic reviews regarding studies until 2012, only studies over the last five years, without language constrictions, were considered. Studies of populations, for whom the subscapularis was the primary goal of surgery, or studies which included healthy controls, were excluded. The target condition was the presence of a partial or full thickness tear of the subscapularis tendon.

\section{Search and Study Selection}

An electronic search strategy in the database of MEDLINE for eligible studies was performed. Reference lists of the publications found were manually checked for additional studies. Two reviewers (MK and EKL) independently screened titles and abstracts of relevant studies for inclusion. They obtained full text of potentially relevant references and determined the inclusion and exclusion criteria of the studies. Uncertainties or disagreements were discussed with a third reviewer (ESTI). In each case a consensus between the two reviewers could be established.

\section{Data Collection}

Two reviewers (MK and EKL) independently collected the available data. When necessary, the study authors were contacted for additional information. For inclusion, data had to be sufficient for creating a $2 \times 2$ table. A number of data was collected, such as the general information of the study (title, authors, journal, year, study design, level of evidence), sample size, patient characteristics, target condition, description of the index and reference test and the number of true positives (TP), true negatives (TN), false positives (FP), and false negatives (FN) of overall tears / partial- and full thickness tears.

\section{Risk of Bias Assessment}

The methodological quality of the included studies was accessed by two reviewers (MK and EKL) using the Quadas-2 checklist [20] (Appendix 1). Disagreement on study quality was resolved by consensus. Methodological quality was plotted in the Review Maker 5.3 (RevMan 5) as provided by the Cochrane Community [21].

\section{Data Analysis}

The accuracy of the index test for each target condition was estimated in sensitivity and specificity with $95 \%$ confidence intervals. The result of each study was shown in a forest plot also created with the Review Maker 5.3 (Figure 1).

\section{Results}

\section{Study Selection}

The initial search strategy (Appendix 2) identified a total of 590 studies. The results are shown in the PRISMA flow chart presented in Figure 1. Seventeen articles were selected for full review, while 155 were excluded by consensus. Of these 17, four were considered in this systematic review. A total of 13 articles were excluded.

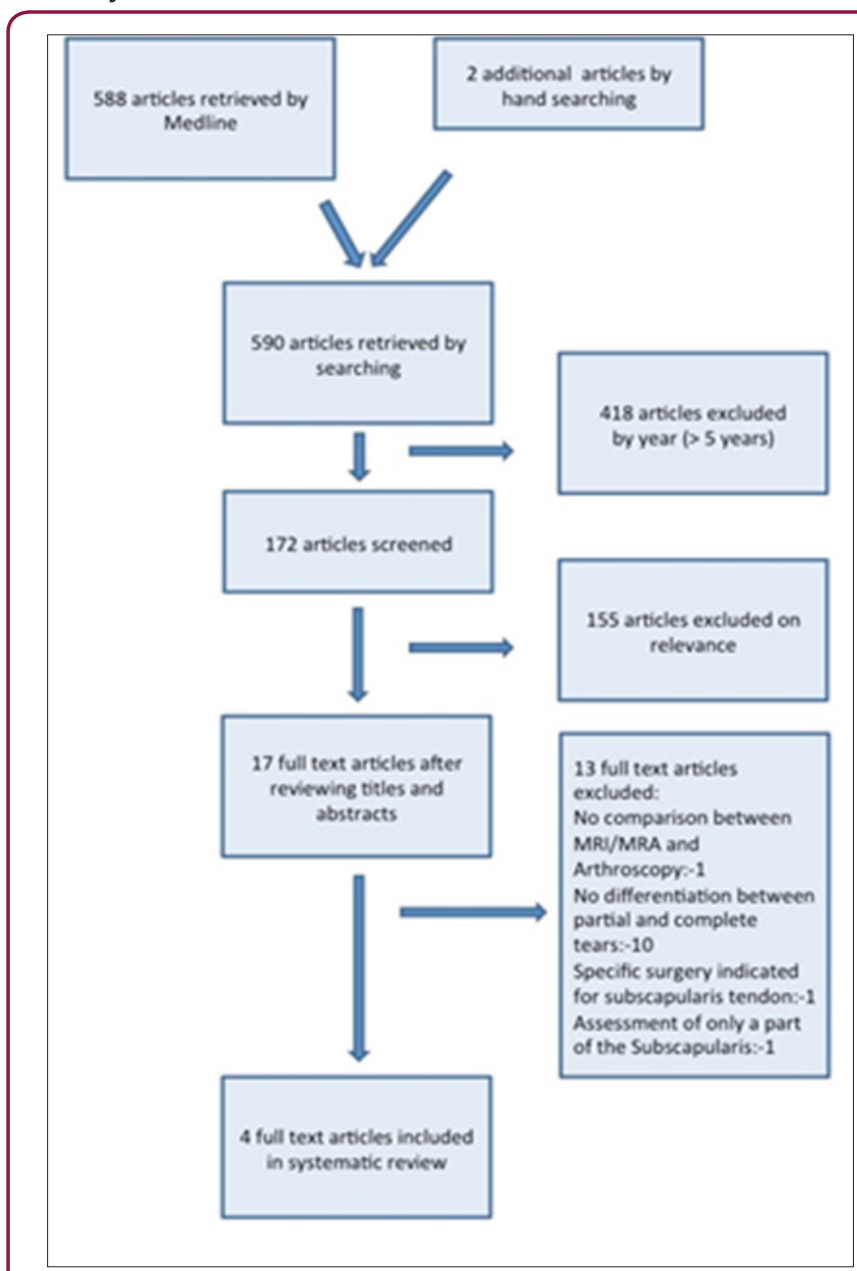

Figure 1: Study flow diagram for identification of included studies.

\section{Risk of Bias}

The methodological quality of the four included studies was judged by two reviewers (MK and EKL). They assessed the risk of bias to be high, low or unclear in different categories. Uncertainties or disagreements were discussed with a third reviewer (ESTI) until a consensus was made. The result for all studies is summarised in Figure 2. The result for the individual studies can be found in Figure 3. Patient selection created a high risk of bias in two studies because of rigorous exclusion criteria [10] or exclusions based on lack of information about surgical findings [22]. Bias on the index test was based on lack of reporting definition and threshold of the target condition [22]. Applicability concerns of the index test were based on a lack of making a difference between partial and full tear $[23,24]$. Arthroscopy was the reference standard in all studies. Although not mentioned in all studies, it can be assumed that - 
based on ethical reasons - the surgeons were aware of the results of the imaging tests before surgery. In one study [22] the definition and threshold for the target condition in the reference test was not mentioned. The time interval between index and reference test was not mentioned in one [22], less than 3 months in one [23] and less than 6 months in two studies $[10,24]$.

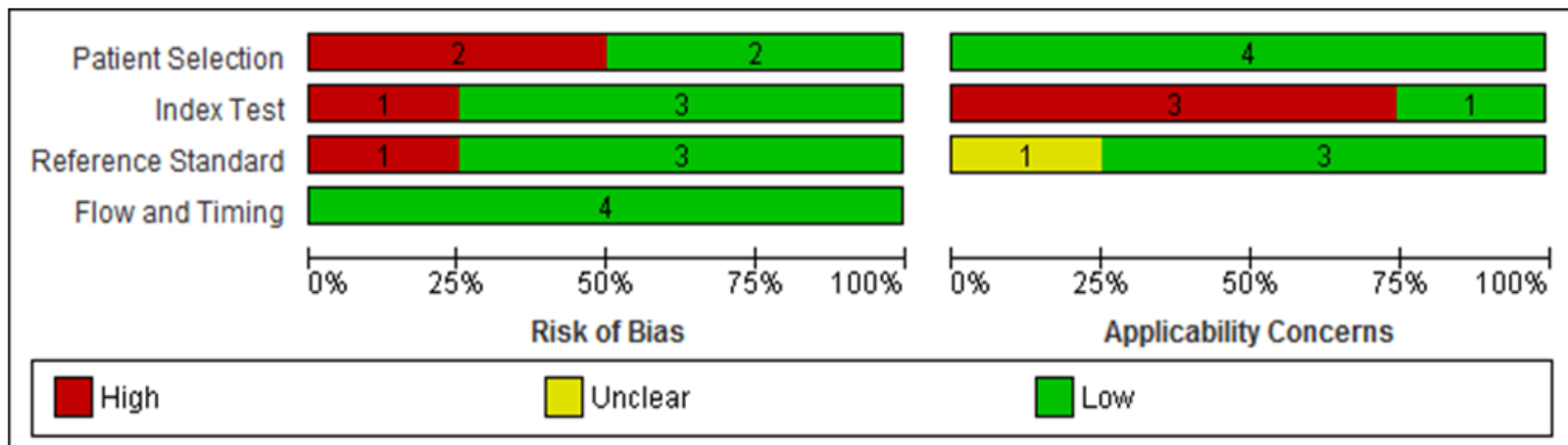

Figure 2: Risk of bias and applicability concerns graph: review author's judgment about each domain presented as percentages across included studies.

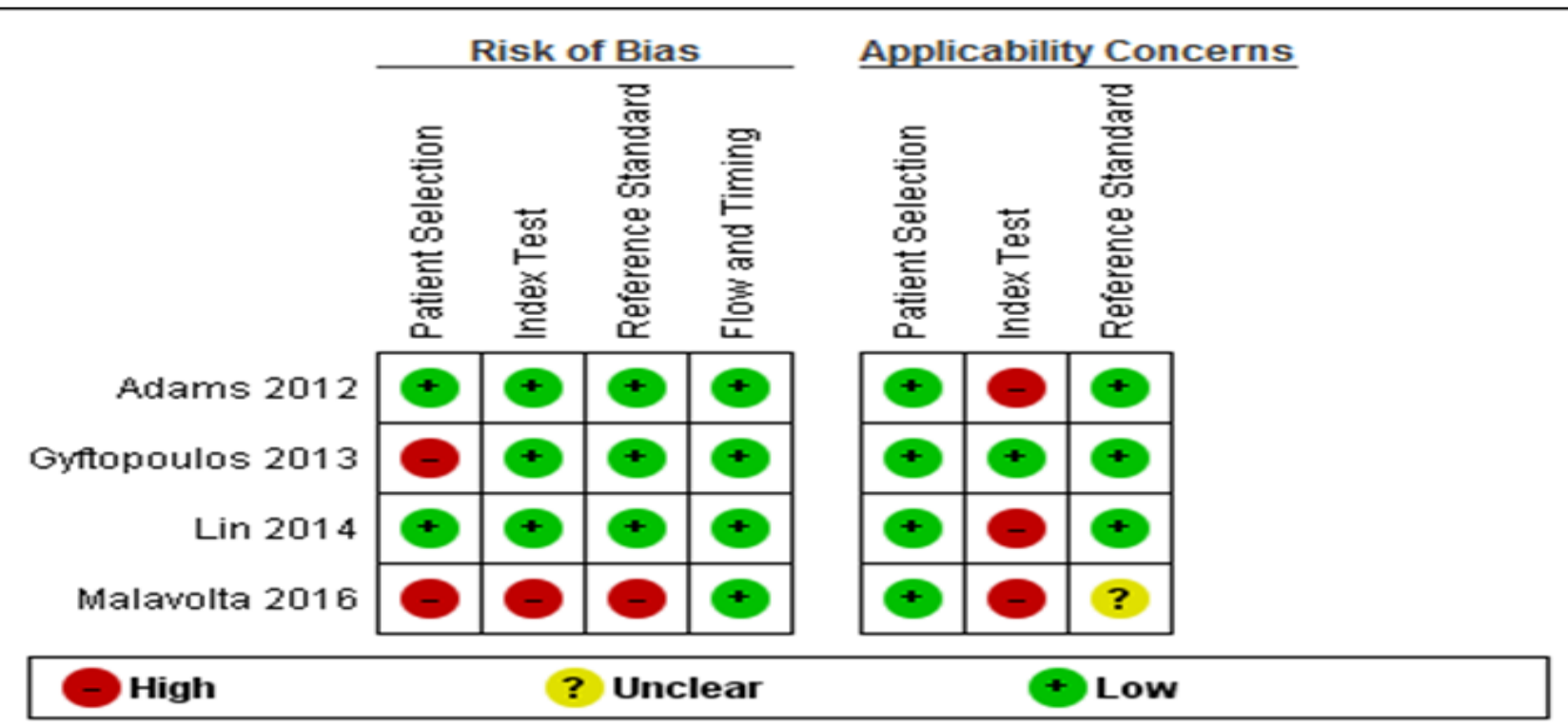

Figure 3: Risk of bias applicability concerns summary: review authors' judgment about each domain for each included study.

\section{Characteristics of the Included Studies}

All four studies compared retrospectively arthroscopic findings to prior made MRI/MRA findings. The arthroscopic findings were divided in full thickness, partial thickness, or no tears of the subscapularis tendon. The MRI findings in two studies [10,22] also divided in full thickness, partial thickness and no tears. The other two studies $[23,24]$ described different tear signs and made a cutoff point between tear and no tear.

\section{Detection of Subscapularis Tears}

Figure 4 shows the forest plots of sensitivity and specificity for MRI that assessed any tear of the subscapularis tendon. The four studies based on 811 participants, having 264 subscapularis tears show a mean prevalence of $32.6 \%$ (range $10.2 \%$ to $53.7 \%$ ). The sensitivity of MRI reported in the studies varied from $61 \%$ to $82 \%$, and specificity from $86 \%$ to $96 \%$ (Figures $5 \& 6$ ). In three studies [22-24] no difference in tear size of the MRI findings were made. As a result, it was only possible to evaluate if an arthroscopic found (partial- or full thickness) tear was detected or not (Figure 6). Partial thickness tears were present in 221 of 811 cases (27.3\%). The sensitivity for detecting partial thickness tears with MRI varied from $54 \%$ to $81 \%$ (Figure 5). Only the study of Gyftopoulos et al. [10] described the possibility to correctly rule out a partial tear by MRI. The specificity in this study was 92\% (0.88-0.95 95\%CI). Full thickness tears were present in 43 of 811 cases $(5,3 \%)$. The sensitivity for detecting full thickness tears with MRI varied from 93 to $100 \%$ (Figure 6). The study of Gyftopoulos et al. [10] described a specificity of MRI to correctly rule out a full thickness tear of $100 \%$ (0.98- $1.0095 \% \mathrm{CI})$. 
MRI vs Athroscopy

\begin{tabular}{|c|c|c|c|c|c|c|c|}
\hline Study & TP & FP & FN TN & Sensitivity $(95 \% \mathrm{Cl})$ & Specificity $(95 \% \mathrm{Cl})$ & Sensitivity $(95 \% \mathrm{Cl})$ & Specificity $(95 \% \mathrm{Cl})$ \\
\hline Adams 2012 & 50 & 5 & 32115 & $0.61[0.50,0.72]$ & $0.96[0.91,0.99]$ & - & \\
\hline Gyftopoulos 2013 & 20 & 19 & 5200 & $0.80[0.59,0.93]$ & $0.91[0.87,0.95]$ & $\because-$ & \\
\hline Lin 2014 & 88 & 13 & 19152 & $0.82[0.74,0.89]$ & $0.92[0.87,0.96]$ & - & \\
\hline Malavolta 2016 & 39 & 6 & 1137 & $0.78[0.64,0.88]$ & $0.86[0.72,0.95]$ & $\rightarrow$ & +7 \\
\hline
\end{tabular}

Figure 4: Studies evaluating sensitivity and specificity in detecting subscapularis tears by MRI compared to arthroscopic findings as a gold standard in four studies (forest plot).

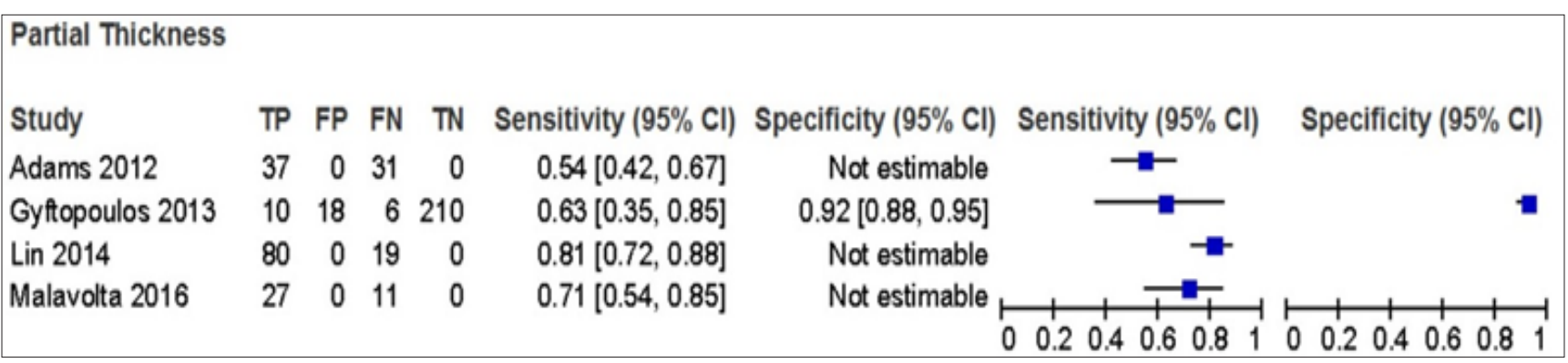

Figure 5: Sensitivity of a partial tear of the subscapularis tendon by MRI compared to arthroscopic findings as a gold standard in four studies (forest plot).

\begin{tabular}{|c|c|c|c|c|c|c|c|c|}
\hline \multicolumn{9}{|l|}{ Full Thickness } \\
\hline Study & TP & FP & FN & $\mathrm{TN}$ & Sensitivity $(95 \% \mathrm{Cl})$ & Specificity (95\% Cl) & Sensitivity $(95 \% \mathrm{Cl})$ & Specificity $(95 \% \mathrm{Cl})$ \\
\hline Adams 2012 & 13 & 0 & 1 & 0 & $0.93[0.66,1.00]$ & Not estimable & $\longrightarrow$ & \\
\hline Gyftopoulos 2013 & 9 & 1 & 0 & 234 & $1.00[0.66,1.00]$ & $1.00[0.98,1.00]$ & & \\
\hline Lin 2014 & 8 & 0 & 0 & 0 & $1.00[0.63,1.00]$ & Not estimable & & \\
\hline Malavolta 2016 & 12 & 0 & 0 & 0 & $1.00[0.74,1.00]$ & Not estimable & 1 & \\
\hline
\end{tabular}

Figure 6: Sensitivity of detecting a full thickness of the subscapularis tendon by MRI compared to arthroscopic findings as a gold standard in four studies (forest plot).

\section{Discussion}

This review summarizes the evidence for the diagnostic accuracy of MRI/MRA of detecting subscapularis tears in patients undergoing rotator cuff surgery in the last five years. Among 811 patients there were 451 male and 360 female, they had a mean age of 51.5 years (47.9-56.2). A good sensitivity for detecting full thickness tears, but only a poor to moderate sensitivity in detecting partial thickness tears of the subscapularis tendon was found. Considering the retrospective character of the included studies and the knowledge that these studies produce spectrum and verification bias and tend to yield falsely inflated sensitives [25], the effective sensitivity is likely to be even lower. Although all studies were performed on modern high end MRI 1.5T and/or 3.0T scans, these findings did not differ from previous reviews on this topic $[3,15,17]$. The clinical consequences of these findings are not to underestimate, as it seems to be necessary to repair subscapularis tears $\geq 5 \mathrm{~mm}(=20 \%)$ [22].
All four studies included in this review showed that there is still no consensus in how to diagnose and classify subscapularis tears in MRI as well as in arthroscopy. Two studies [23,24] diagnosed subscapularis tears in MRI based on seven, resp. four direct and indirect signs. The cut off for defining a tear was 2 out of 7 (without explanation), resp. 2 out of 4 based on a pilot study. For the arthroscopy, one study used the classification by Lafosse et al. [26]. \& Kim et al.[27], while the other study described the size of tear in percentage. In one study [10] the radiologist and the surgeon could both define between "no tear", "partial thickness tear", "full thickness, complete tear" and "full thickness, incomplete tear" but in the results no distinction was found between "complete" and "incomplete" tear. The fourth study [22] made a difference between "no tear", "partial tear" and "full thickness tear" in MRI and arthroscopy, but did not describe on what findings these distinctions were made. Lack of consensus leads to a high level of heterogeneity in the field of diagnostic test accuracy studies. 
This makes it impossible to make a direct comparison and a metaanalysis.

The study of Gyftopoulos et al. [10] stood out because of a low prevalence $(10 \%)$ of subscapularis tears in patients who underwent arthroscopy for rotator cuff injuries. By having a closer look, two major problems were found. Patients who underwent MRA $(n=32)$ were excluded. Especially patients with suspected tears would have additional MRA diagnostic, which might have had consequences on the prevalence: for example $18.3 \%$ of the included patients in the study of Adams et al. [24] also had a MRA. Secondly, retrospectively tears of the inferior tendon were excluded and intratendinous tears, as well as bursal-sided tears were documented as intact or normal tendons. These restrictions might have reduced false positive findings and improved specificity in this study.

\section{Limitations of this Review}

The purpose of this review was to conduct a quantitative result for detecting partial- and full thickness tears of the subscapularis tendon in patients scheduled for rotator cuff surgery. A search strategy in only one database and a rigorous exclusion of literature resulted in a mini-review of just 4 articles. In addition, due to the retrospective character and a high level of heterogeneity in these studies, it was impossible to pool data and makes a quantitative statement.

\section{Conclusion}

Despite the technological improvements in the last five years the sensitivity of detecting subscapularis tears in patients scheduled for rotator cuff surgery remains challenging. Because the result of rotator cuff surgery depends on the integrity of the subscapularis tendon, surgeons need to be aware of possible subscapularis tears although MRI/MRA scans may be negative. A consensus on how to classify subscapularis tears in MRI/MRA and arthroscopy findings might be helpful for diagnostic and would enable to perform more qualitative (prospective) research.

\section{References}

1. Ticker JB, Warner JJP (1997) Single-tendon tears of the rotator cuff: Evaluation and treatment of subscapularis tears and principles of treatment for supraspinatus tears. Orthop Clin North Am 28(1): 99-116.

2. Shi LL, Mullen MG, Freehill MT, Lin A, Warner JJP, et al. (2015) Accuracy of long head of the biceps subluxation as a predictor for subscapularis tears. Arthroscopy 31(4): 615-619.

3. Lenza M, Buchbinder R, Takwoingi Y, Johnston RV, Hanchard NC, et al. (2013) Magnetic resonance imaging, magnetic resonance arthrography and ultrasonography for assessing rotator cuff tears in people with shoulder pain for whom surgery is being considered. Cochrane Database Syst Rev 24(9).

4. Sakurai G, Ozaki J, Tomita Y, Kondo T, Tamai S (1998) Incomplete tears of the subscapularis tendon associated with tears of the supraspinatus tendon: cadaveric and clinical studies. J Shoulder Elbow Surg 7(5): 510515 .

5. Takeda Y, Fujii K, Miyatake K, Kawasaki Y, Nakayama T, et al. (2016) Diagnostic Value of the Supine Napoleon Test for Subscapularis Tendon Lesions. Arthrosc - J Arthrosc Relat Surg 32(12): 2459-2465.

6. Barth JRH, Burkhart SS, De Beer JF (2006) The Bear-Hug Test: A New and Sensitive Test for Diagnosing a Subscapularis Tear. Arthrosc - J Arthrosc Relat Surg 22(10): 1076-1084.
7. Garavaglia G, Taverna E, Ufenast H (2011) The frequency of subscapularis tears in arthroscopic rotator cuff repairs: A retrospective study comparing magnetic resonance imaging and arthroscopic findings. Int J Shoulder Surg 5(4): 90.

8. Rigsby R, Sitler M, Kelly JD (2010) Subscapularis tendon integrity: An examination of shoulder index tests. J Athl Train.

9. Warner JJ, Higgins L, Parsons IM, Dowdy P (1996) Diagnosis and treatment of anterosuperior rotator cuff tears. J Shoulder Elbow Surg 10(1): 37-46.

10. Gyftopoulos S, O’Donnell J, Shah NP, Goss J, Babb J, et al. (2013) Correlation of MRI with arthroscopy for the evaluation of the subscapularis tendon: A musculoskeletal division's experience. Skeletal Radiol 42(9): 12691275.

11. Jung JY, Jee WH, Chun CW, Kim YS (2017) Diagnostic Performance of MR Arthrography with Anterior Trans-Subscapularis versus Posterior Injection Approach for Subscapularis Tendon Tears at 3.0T. Eur Radiol 27(3): 1303-1311.

12. Arai R, Sugaya H, Mochizuki T, Nimura A, Moriishi J, et al. (2008) Subscapularis Tendon Tear: An Anatomic and Clinical Investigation. Arthrosc - J Arthrosc Relat Surg 24(9): 997-1004.

13. Boileau P (2005) Arthroscopic Repair of Full-Thickness Tears of the Supraspinatus: Does the Tendon Really Heal? J Bone Jt Surg 87(6): 1229.

14. Ide J, Tokiyoshi A, Hirose J, Mizuta H (2007) Arthroscopic repair of traumatic combined rotator cuff tears involving the subscapularis tendon. J Bone Joint Surg Am 89(11): 2378-2388.

15. Roy JS, Braën C, Leblond J, Desmeules F, Dionne CE, et al. (2015) Diagnostic accuracy of ultrasonography, MRI and MR arthrography in the characterisation of rotator cuff disorders: a systematic review and meta-analysis. Br J Sports Med 49(20): 1316-1328.

16. De Jesus JO, Parker L, Frangos AJ, Nazarian LN (2009) Accuracy of MRI, MR arthrography, and ultrasound in the diagnosis of rotator cuff tears: A meta-analysis. Am J Roentgenol 192(6): 1701-1707.

17. Schultz W, M Von Knoch (2014) Darstellung von Rissen der Subscapularissehne im Vergleich. Orthopädische und Unfallchirurgische Prax 3(10): 466-469.

18. Foad A, Wijdicks CA (2012) the accuracy of magnetic resonance imaging and magnetic resonance arthrogram versus arthroscopy in the diagnosis of subscapularis tendon injury. Arthrosc - J Arthrosc Relat Surg 28(5): 636-641.

19. Shapiro L, Harish M, Hargreaves B, Staroswiecki E, Gold G (2012) Advances in musculoskeletal MRI: Technical considerations. J Magn Reson Imaging 36(4): 775-787.

20. Whiting PF, Rutjes AWS, Westwood ME, Mallett S, Deeks JJ, et al. (2011) QUADAS-2: a revised tool for the quality assessment of diagnostic accuracy studies. Ann Intern Med 155(4): 529-536.

21. Cochrane Community. RevMan p. 5.

22. Malavolta EA, Assunção JH, Guglielmetti CLB, De Souza FF, Gracitelli $\mathrm{ME}$, et al. (2016) Accuracy of preoperative MRI in the diagnosis of subscapularis tears. Arch Orthop Trauma Surg 136(10): 1425-1430.

23. Lin L, Yan H, Xiao J, He Z, Luo H, et al. (2014) The diagnostic value of magnetic resonance imaging for different types of subscapularis lesions. Knee Surgery, Sport Traumatol Arthrosc 24(7): 2252-2258.

24. Adams CR, Brady PC, Koo SS, Narbona P, Arrigoni P, et al. (2012) A systematic approach for diagnosing subscapularis tendon tears with preoperative magnetic resonance imaging scans. Arthrosc - J Arthrosc Relat Surg 28(11): 1592-1600.

25. Naimark M, Zhang AL, Leon I, Trivellas A, Feeley BT, et al. (2016) Clinical, Radiographic, and Surgical Presentation of Subscapularis Tendon Tears: A Retrospective Analysis of 139 Patients. Arthrosc. J Arthrosc Relat Surg 32(5): 747-752. 
26. Lafosse L, Jost B, Reiland Y, Audebert S, Toussaint B, et al. (2007) Structural Integrity and Clinical Outcomes After Arthroscopic Repair of Isolated Subscapularis Tears. J Bone Jt Surg 89(6).
27. Kim TK, Rauh PB, McFarland EG Partial tears of the subscapularis tendon found during arthroscopic procedures on the shoulder: a statistical analysis of sixty cases. Am J Sports Med 31(5): 744-750.

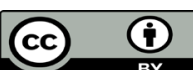

This work is licensed under Creative Commons Attribution 4.0 License

Submission Link: http://biomedres.us/submit-manuscript.php

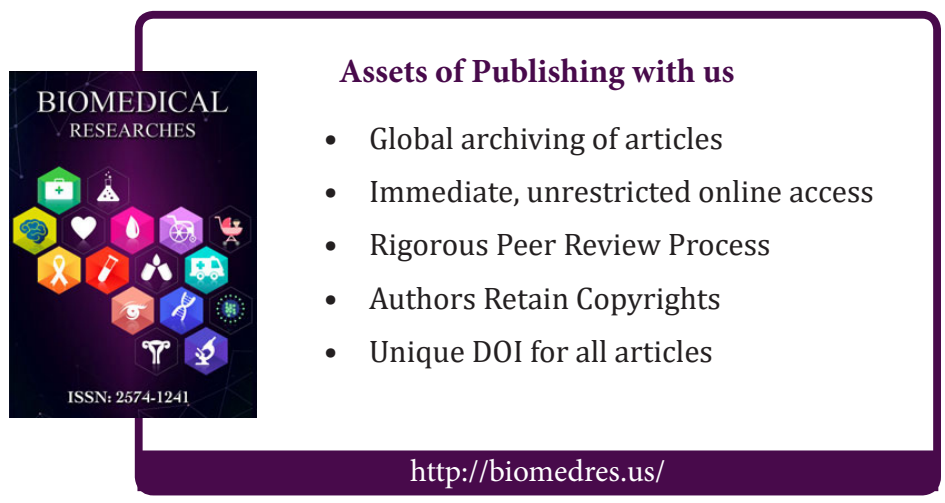

